\title{
Linear confinement of a scalar and spin-0 particle in a topologically trivial flat Gödel-type space-time
}

\author{
Faizuddin Ahmed ${ }^{\mathrm{a}}$ \\ Ajmal College of Arts and Science, Dhubri, Assam 783324, India
}

Received: 6 August 2018 / Accepted: 21 January 2019 / Published online: 1 February 2019

(C) The Author(s) 2019

\begin{abstract}
In the previous work (Ahmed, Eur Phys J C 78(7):598, 2018), we investigated the relativistic quantum effects on a scalar and spin-half particles in a topologically trivial flat Gödel-type space-time. We have found that the energy eigenvalues of the system are influenced by the vorticity parameter characterizing the space-time. In the present work, we investigate the linear confinement of a scalar particle on the Klein-Gordon equation with a linear and Coulombtype scalar potential in this flat Gödel-type solution. The energy eigenvalues of the system get modifies due to the presence of scalar potentials, and the vorticity parameter. In addition, we study the relativistic quantum motion of spin- 0 particles with vector and scalar potentials of Coulomb-type and analyze the effects on the energy eigenvalues.
\end{abstract}

\section{Introduction}

The first solution to the Einstein's field equations containing closed time-like curves is the cylindrical symmetry Gödel rotating Universe [2]. After that, a variety of solutions admitting closed time-like curves, closed null geodesics and closed time-like geodesics has been constructed in general relativity (see $[3,4]$ and references therein). To prevent the appearence of closed time-like curves in a space-time, Hawking [5] proposed the Chronology Protection Conjecture. However, the general proof of this Conjecture has not yet been given. On the contrary, there are many solutions of the field equations in vacuum as well as non-vacuum known that contains such closed causal curves. Of these, some solutions with closed time-like curves content exotic matter-energy sources violating so called the weak energy condition (WEC) (e.g. [6,7]) and some others violate the strong energy condition (e.g. [8-10]). Reboucas et al. [11-13] investigated the Gödel-type solutions characterized by vorticity, which represents a generalization of the original Gödel metric with possible sources

a e-mail: faizuddinahmed15@gamil.com and analyzed the problem of causality. The general Gödeltype metrics in the presence of cosmic strings in polar coordinates $(t, r, \phi, z)$ can be written as

$d s^{2}=-(d t+\alpha \Omega F(r) d \phi)^{2}+\alpha^{2} H^{2}(r) d \phi^{2}+d r^{2}+d z^{2}$,

where the metric functions are

$F(r)=\frac{\sinh ^{2} l r}{l^{2}}, \quad H(r)=\frac{\sinh 2 l r}{2 l}$.

The variables $(t, r, \phi, z)$ can take, respectively the values : $-\infty<(t, z)<\infty, 0 \leq r, \phi \in[0,2 \pi]$. The parameter $\Omega$ characterizes the vorticity of the space-time. The original Gödel solution [2] can be recovered for $l^{2}=\frac{\Omega^{2}}{2}$ and disclination parametr $\alpha=1$. Also for $l^{2}=\frac{\Omega^{2}}{2}$ and $\alpha=1$, one will obtain an anti-de Sitter space [14]. In the asymptotic limit $l \rightarrow 0$, the metric (1) reduces to the well-known Som-Raychaudhuri space-time [15], which is also called flat Gödel-type metric with cosmic string. So the metric in this condition is reduced to

$d s^{2}=-\left(d t+\alpha \Omega r^{2} d \phi\right)^{2}+\alpha^{2} r^{2} d \phi^{2}+d r^{2}+d z^{2}$.

This solution attracted much attention in string theory [1620] and it has interpreted as a Gödel-type solution in string theory. Observe that the line element defined above can be written as

$d s^{2}=-\left(d t+A_{i} d x^{i}\right)^{2}+h_{i j} d x^{i} d x^{j}$,

where the spatial coordinates of the space-time are represented by $x^{i}$. An interesting property of this metric is that the geodesics are circles, which have a physical description similar to Larmor orbits for an electron that moves in a perpendicular magnetic field [21,22]. This analogy also arises 
from the point of view of quantum mechanics, where the quantum dynamics of scalar and spinorial quantum particles in this space-time is an analogue of the Landau levels, as observed in [21-25]. Several researchers have studied the physical properties of a series of backgrounds with the SomRaychaudhuri space-time. For example, Paiva et al. have investigated the properties of the rotating Som-Raychaudhuri homogeneous space-time [26], Wang et al. [27] have studied the relativistic quantum dynamics of a spinless particle and the Klein-Gordon oscillator in the Som-Raychaudhuri space-time under the influence of the gravitational field produced by a topology, Carvalho et al. [23] have worked about the Klein-Gordon oscillator in Som-Raychaudhuri spacetime with a cosmic disclination, and Vitoria et al. [28] have investigated the Linear confinement of a scalar particle in the Som-Raychaudhuri space-time in the presence of a topological defect. They analyzed the influence of topology of the cosmic string and the vorticity parameter on the relativistic energy eigenvalues and found that the energy eigenvalues get modified.

Investigation of relativistic quantum effects on scalar and spin-half particles in Gödel as well as Gödel-type spacetimes have been addressed by several authors (see [1] and references therein). Soares et al. [29] first studied this problem, where the Klein-Gordon and Dirac equations in a class of Gödel-type space-times with positive and negative curvatures, were investigated and also in flat Gödel-type spacetime. Drukker et al. [21] have investigated the close relationship between the quantum dynamics of a scalar particle in background of general relativity with Gödel solutions and the Landau levels in the flat, spherical and hyperbolic spaces. They solved the Klein-Gordon equation in these class of Gödel-type space-times and observed the similarity of the energy eigenvalues with the Landau levels in curved background (see also [30]). Das et al. [22] also have investigated the same problem by studying the Klein-Gordon equation in flat Gödel-type solution (called Som-Raychaudhuri spacetime) and Landau levels in flat spaces. Furtado et al. [31] have studied the Landau levels in the presence of disclination parameter. Carvalo et al. [23] solved the Klein-Gordon equation in a class of Gödel-type solutions with a cosmic string and analyzed the similarity of the energy eigenvalues with the Landau levels in flat, spherical and hyperbolic spaces. They demonstrated there that the presence of a cosmic string, and the vorticity parameter modifies the energy levels and breaks the degeneracy of energy eigenvalues. The quantum influence of topological defects in Gödel-type space-times in flat, spherical and hyperbolic cases, were investigated in [24]. The relativistic quantum dynamics of Dirac particle with the topological defect in a class of Gödel-type space-times with torsion have been investigated in [25]. In Ref. [32] (see also $[33,34]$ ), Weyl fermions in a class of family of Gödel-type geometries with a topological defect, were investigated. In
Ref. [35], relativistic wave equation for spin-half particles in the Melvin space-time, a space-time where the metric is determined by a magnetic field, were investigated.

The relativistic quantum dynamics of a scalar particle subject to different confining potentials has been investigated in several areas of physics by various authors [3650]. The linear confinement of quantum particles has great importance for models of confinement of quarks [51]. It is worth mentioning the linear scalar potential has attracted a great interest in atomic and molecular physics [52-58] and also in relativistic quantum mechanics [59-79]. Another important case is the confinement of a scalar particle subjected to Coulomb-type potential which were investigated by many authors [80-84]. It is worth mentioning studies that have dealt with Coulomb-type potential in the propagation of gravitational waves [85], quark models [51], and relativistic quantum mechanics [86-89]. In Refs. [84,90], a scalar potential is introduced (non-electromagnetic potential) in the Klein-Gordon equation by making a modification in the mass term in the form : $m \rightarrow m+S$, where $m$ is the mass of the free particle and $S$ is the scalar potential (linear or Coulomb-type). This modification in the mass term has been explored in recent decades, for instance, by analysing the behaviour of Dirac particles in the presence of a static scalar and Coulomb potential [91], relativistic scalar particle in the presence of a cosmic string [92]. The quark-antiquark interaction is mapped into a problem of relativistic spinless possessing a position-dependent mass (PDM), where the mass term acquires a contribution given by the interaction potential that consist of linear and harmonic confining potential plus a Coulomb potential term, were investigated in [93]. The Klein-Gordon equation with vector and scalar potentials of Coulomb-types under the influence of non-inertial effects in the cosmic string space-time, were studied by Santos et al. [94]. The relativistic quantum effects of confining potentials on the Klein-Gordon oscillator, were investigated in [77]. Boumali et al. [95] investigated the Klein-Gordon oscillator in the background of cosmic strings in the presence of a uniform magnetic field. Later, the Klein-Gordon oscillator was investigated in the presence of Coulomb-type potential by two ways: (1) via a modification of mass term [89], and (2) via the minimal coupling [78], in the latter case the linear scalar potential was also included. The Klein-Gordon oscillator in curved background within the Kaluza-Klein theory, were investigated in [24]. Recently, Santos et al. [94] investigated the Klein-Gordon oscillator in the background spacetime generated by a cosmic string. A scalar quantum particle confined in two concentric thin shells in curved space-time backgrounds with a cosmic string, were investigated in [33]. It is worth mentioning the studies of Dirac oscillator under the influence of non-inertial effects in the background of cosmic string space-time [96], and the rotating effects in the cosmic string space-time [97]. In Ref. [98], two different classes of 
solution for the Klein-Gordon equation in the presence of a scalar potential under the influence of noninertial effects in the cosmic string space-time, were discussed. The behaviour of a scalar particle within the Yukawa-like potential in SomRaychaudhuri space-time, were investigated in [99].

\section{The Klein-Gordon and spin-0 Equation}

The relativistic quantum dynamics of a free spinless particle of mass $m$ is described by the Klein-Gordon equation. In its covariant form, this equation takes the following form:

$\frac{1}{\sqrt{-g}} \partial_{\mu}\left(\sqrt{-g} g^{\mu \nu} \partial_{\nu} \Psi\right)=m^{2} \Psi, \quad \mu, v=0,1,2,3$,

with $g=\operatorname{det}\left(g_{\mu \nu}\right)$ being the determinant of metric tensor with inverse $g^{\mu \nu}$, and $\partial_{\mu}$ is the ordinary derivative. The function $\Psi$ gives the amplitude of the probability to find the particles around the position $r$ at the time $t$. By introducing a scalar potential into the Klein-Gordon equation by modifying the mass term in the form : $m \rightarrow m+S$, where $S$ is the scalar potential $[84,90]$. The KG-equation becomes

$\frac{1}{\sqrt{-g}} \partial_{\mu}\left(\sqrt{-g} g^{\mu \nu} \partial_{\nu} \Psi\right)=(m+S)^{2} \Psi$.

The spin-0 particles are represented by the usual KleinGordon equation which can be generalized to the curved space-time case. In order to determine the generalization of the wave equation one may replace the ordinary derivatives by covariant derivative [98] in the Klein-Gordon equation, the result is

$$
\frac{1}{\sqrt{-g}} D_{\mu}\left(\sqrt{-g} g^{\mu \nu} D_{\nu} \Psi\right)=m^{2} \Psi
$$

that is, the Klein-Gordon equation in a curved space-time [100] where, $D_{\mu}=\partial_{\mu}-i e A_{\mu}, e$ is the electric charge, and $A_{\mu}$ is the vector potential. A scalar potential $S$ may be taken into account by making a modification on the mass term: $m \rightarrow m+S$. Substituting this mass term into Eq. (7) we obtain the following differential equation:

$$
\frac{1}{\sqrt{-g}} D_{\mu}\left(\sqrt{-g} g^{\mu \nu} D_{\nu} \Psi\right)=(m+S)^{2} \Psi
$$

This differential equation takes into account a scalar potential $S$ and vector potential $A_{\mu}[83,88,101]$. In order to simplify solution of the Klein-Gordon equation, the four-vector potential can be written as $A_{\mu}=\left(A_{0}, 0,0,0\right)$. The first component of the four-vector potential is represented by a vector potential, i.e., $A_{0}=V$. To have a bound-state (real) solutions for a relativistic spin-zero particle, the relationship between vector and scalar potential must be $S \geq V$ [38,42-44].
In the present work, we consider a flat Gödel-type spacetime and discuss the linear confinement of a relativistic scalar particle with a linear scalar potential. We also study solution of the Klein-Gordon equation in the presence of Coulombtype scalar potential and analyze the influence of confining potential, and the vorticity parameter on the energy eigenvalues. In addition, we investigate the relativistic quantum motion of spin- 0 particles with scalar and vector potentials of Coulomb-type and analyze the effects.

\section{A flat Gödel-type space-time}

Consider the following stationary space-time [102] (see also [1]) in the coordinates $\left(x^{0}=t, x^{1}=x, x^{2}=y, x^{3}=z\right)$ given by

$$
\begin{aligned}
d s^{2} & =-d t^{2}+d x^{2}+\left(1-\alpha^{2} x^{2}\right) d y^{2}-2 \alpha x d t d y+d z^{2} \\
& =-(d t+H(x) d y)^{2}+d x^{2}+D^{2}(x) d y^{2}+d z^{2},
\end{aligned}
$$

where $\alpha>0$ is a real number, and the function $H(x)=\alpha x$ and $D(x)=1$. The ranges of the coordinates are $-\infty<$ $(t, x, y, z)<\infty$. By choosing periodicity of the $y$ coordinate, one can easily show that the space-time display causality violation, namely, closed time-like curves for $x>\frac{1}{\alpha}$ and closed null curve at $x=x_{0}=\frac{1}{\alpha}$. The determinat of the metric tensor for the metric (9) is detg $=-1$ and the covariant and contravariant components of the metric tensor are

$g_{00}=-1, \quad g_{11}=g_{33}=1, \quad g_{02}=-\alpha x=g^{02}, \quad g^{11}=1$, $g_{22}=\left(1-\alpha^{2} x^{2}\right), g^{00}=-\left(1-\alpha^{2} x^{2}\right), g^{22}=g^{33}=1$.

And that the Christoffel symbols are

$\Gamma_{01}^{0}=\frac{1}{2} \alpha^{2} x=-\Gamma_{12}^{2}=\frac{1}{2} \Gamma_{22}^{1}$,

$\Gamma_{12}^{0}=\frac{1}{2} \alpha\left(1+\alpha^{2} x^{2}\right), \quad \Gamma_{02}^{1}=\frac{1}{2} \alpha=-\Gamma_{01}^{2}$.

The necessary and sufficient conditions for Gödel-type space-times to be space-time homogeneous (STH) [11,103105] are given by

$\frac{H^{\prime}}{D}=\alpha=2 \Omega, \quad \frac{D^{\prime \prime}}{D}=0$

where prime denotes ordinary differentiation w. r. t. $x$. Here $\Omega$ is the vorticity parameter characterising the space-time.

The metric (9) by substituting $\alpha=2 \Omega$ can be expressed in the form (4) as:

$d s^{2}=-(d t+2 \Omega x d y)^{2}+\delta_{i j} d x^{i} d x^{j}$, 
where the vector potential $A_{y}=2 \Omega x$, and $\delta_{i j}$ is the kronecker delta. The corresponding uniform magnetic field associated with the vector potential is along $z$-axis given by $B_{z}=2 \Omega$.

In cartesian coordinates system, the Som-Raychaudhuri space-time (3) can be written as

$d s_{S R}^{2}=-[d t+\Omega(x d y-y d x)]^{2}+\delta_{i j} d x^{i} d x^{j}$,

by transforming $r \rightarrow \sqrt{x^{2}+y^{2}}$ and $\phi \rightarrow \tan ^{-1}\left(\frac{y}{x}\right)$ into (3). In that case, the vector potentials are $A_{x}=-\Omega y, A_{y}=\Omega x$ and the corresponding magnetic field is along $z$-axis given by $B_{z}=2 \Omega$.

Note that if one takes $\Omega=0$ which implies $\alpha=0$, the study space-time (9) or (13) reduces to four-dimensional Minkowski flat space metric.

\subsection{Scalar particle with linear and Coulomb-type scalar potentials in flat Gödel-type metric}

For the line element (9), the KG-equation (6) can be written in the following form:

$\left[-\partial_{t}^{2}+\left(\alpha x \partial_{t}-\partial_{y}\right)^{2}+\left(\partial_{x}^{2}+\partial_{z}^{2}\right)\right] \Psi=(m+S)^{2} \Psi$,

which is independent of $t, y, z$. One can choose the following ansatz for the function $\Psi$

$\Psi(t, x, y, z)=e^{i\left(-E t+p_{y} y+p_{z} z\right)} \psi(x)$,

where $E, p_{y}$ and $p_{z}$ are constants. Here we have considered the potentials as follow:

Case A: The linear scalar potential $[84,90]$ is given by

$S(x)=k_{L} x$,

where $k_{L}$ is a constant that characterizes the linear confining potential. Substituting the ansatz given by Eq. (16) into Eq. (15), we obtain the following differential equation for $\psi(x)$ using the potential (17) :

$\psi^{\prime \prime}(x)-\left[\omega^{2} x^{2}+\delta x\right] \psi(x)=\beta \psi(x)$,

where we have written

$\omega=\sqrt{\alpha^{2} E^{2}+k_{L}^{2}}, \quad \delta=2\left(\alpha E p_{y}+m k_{L}\right)$.

Let us define $r=\sqrt{\omega} x$, Eq. (18) becomes

$\psi^{\prime \prime}(r)-\left[r^{2}+\delta_{0} r\right] \psi(r)=\lambda \psi(r)$, where

$\delta_{0}=\frac{\delta}{\omega^{\frac{3}{2}}}, \quad \lambda=\frac{\beta}{\omega}, \quad \beta=\left(p_{y}^{2}+p_{z}^{2}+m^{2}-E^{2}\right)$.

The asymptotic behaviour of the possible solution to the Eq. (20) are to be determined for $r \rightarrow 0$ and $r \rightarrow \infty$. These conditions are necessary since the wave functions must be well-behaved in this limits, and thus, bound states of energy eigenvalues can be obtained. Let us impose that the function $\psi(r)$ is well-behaved at $r \rightarrow 0$ and vanish at $r \rightarrow \infty$, hence, the solution to Eq. (20) is given by

$\psi(r)=e^{-\frac{r^{2}}{2}} e^{-\frac{\delta_{0} r}{2}} H(r)$.

Substituting Eq. (22) into Eq. (20), we obtain the following differential equation

$\frac{d^{2} H}{d r^{2}}-\left[\delta_{0}+2 r\right] \frac{d H}{d r}+\eta H=0$,

where $\eta$ is given by

$\eta=\frac{1}{4} \delta_{0}^{2}-\lambda-1$

Writing the solution as a power series expansion $[31,88,106]$ around the origin :

$H(r)=\sum_{i=0}^{\infty} c_{i} r^{i}$

Substituting the series expansion into the Eq. (23), we obtain the following recurrence relation:

$c_{n+2}=\frac{(2 n-\eta)}{(n+1)(n+2)} c_{n}+\frac{\delta_{0}}{n+2} c_{n+1}$.

By starting with $c_{0}=1$ using Eq. (26), one can calculate other co-efficients of the power series expansion. As examples, the coefficients $c_{2}$ and $c_{3}$ are given by

$c_{2}=\frac{1}{2}\left(\delta_{0} c_{1}-\eta\right), c_{3}=\left(\frac{\delta_{0}^{2}+2-\eta}{6}\right) c_{1}-\frac{\delta_{0} \eta}{6}$.

The power series expansion becomes a polynomial of $r^{n}$ by imposing the following two conditions:

$c_{n+1}=0, \quad \eta=2 n$,

where $n=1,2,3 \ldots$ is the quantum number. By analysing the condition $\eta=2 n$, one can obtain the following energy eigenvalues equation :

$E_{n}^{2}-(2 n+1) \alpha E_{n}-\left(A_{n}+m^{2}+p_{z}^{2}\right)=0$, 
where

$$
\begin{aligned}
A_{n}= & \left(n+\frac{1}{2}\right)\left(1+\frac{k_{L}^{2}}{2 \omega^{2}}\right) \frac{k_{L}^{2}}{\omega}+\left(p_{y}^{2}-m^{2}\right) \frac{k_{L}^{2}}{\omega^{2}} \\
& -2 m k_{L} p_{y}\left(1-\frac{k_{L}^{2}}{2 \omega^{2}}\right), \quad k_{L}<\omega .
\end{aligned}
$$

Therefore the relativistic energy eigenvalues of the system under linear scalar potential are

$$
\begin{aligned}
E_{n} & =\left(n+\frac{1}{2}\right) \alpha+\sqrt{\left(n+\frac{1}{2}\right)^{2} \alpha^{2}+m^{2}+p_{z}^{2}+A_{n}} \\
& =(2 n+1) \Omega+\sqrt{(2 n+1)^{2} \Omega^{2}+m^{2}+p_{z}^{2}+A_{n}}
\end{aligned}
$$

The different coefficients of power series expansion under the condition (28) are

$n=1, \quad c_{2}=0 \Rightarrow c_{1}=\frac{2}{\delta_{0}} c_{0}$

$n=2, \quad c_{3}=0 \Rightarrow c_{1}=\left(\frac{4 \delta_{0}}{\delta_{0}^{2}-2}\right) c_{0}$,

$c_{2}=\left(\frac{4}{\delta_{0}^{2}-2}\right) c_{0}$.

A special case is for $p_{z}=0=m$, we get the following energy eigenvalues of the system under a linear scalar potential

$$
\begin{aligned}
E_{n}= & (2 n+1) \Omega \\
& +\sqrt{(2 n+1)^{2} \Omega^{2}+\left(n+\frac{1}{2}\right)\left(1+\frac{k_{L}^{2}}{2 \omega^{2}}\right) \frac{k_{L}^{2}}{\omega}+\frac{p_{y}^{2} k_{L}^{2}}{\omega^{2}}} .
\end{aligned}
$$

For $k_{L}=0$, that is, no linear scalar confining potential, Eq. (33) the energy eigenvalues of the system are same the energy eigenvalues obtained in [1]. Thus we see that the presence of a linear scalar confining potential, and the vorticity parameter modifies the energy levels of the system. An important note that Eq. (31) the energy eigenvalues for $n^{\text {th }}$ degree polynomial of the system under a linear scalar potential is different from the result obtained in [28]. Even though the considering space-times are of the same cateory, that is, the flat Gödel-type space-times, the result obtained in [28] is for the cylindrical symmetry system whereas the cartesian coordinates system for the present one.

Case B: The scalar potential of Coulomb-type is given by

$S(x)=\frac{k_{c}}{x}$

where $k_{c}$ characterizes the Coulomb-type interaction. Considering the ansatz Eq. (16) into the KG-equation Eq. (15) with potential (34), we obtain the following differential equation for $\psi(x)$ :

$\psi^{\prime \prime}(x)$
$-\left[\alpha^{2} E^{2} x^{2}+\frac{k_{c}^{2}}{x^{2}}+2 \alpha E p_{y} x+\frac{2 m k_{c}}{x}+\beta\right] \psi(x)=0$.

Let us define $r=\sqrt{\alpha E} x$, we have obtained the Schrödinger form

$\psi^{\prime \prime}(r)-\left[r^{2}+\frac{k_{c}^{2}}{r^{2}}+\Theta r+\frac{\xi}{r}+\beta_{0}\right] \psi(r)=0$,

where

$\Theta=\frac{2 p_{y}}{\sqrt{\alpha E}}, \quad \xi=\frac{2 m k_{c}}{\sqrt{\alpha E}}, \quad \beta_{0}=\frac{\beta}{\alpha E}$.

Let the solution of Eq. (36) is

$\psi(r)=r^{\gamma} e^{-\frac{r^{2}}{2}} e^{-\frac{\Theta r}{2}} H(r), \gamma=\frac{1}{2}\left[1+\sqrt{1+4 k_{c}^{2}}\right]$.

Substituting Eq. (38) into Eq. (36), we obtained the following second order differential equation :

$$
\begin{aligned}
& r H^{\prime \prime}(r)+\left[2 \gamma-\Theta r-2 r^{2}\right] H^{\prime}(r) \\
& \quad+\left[r\left(\frac{\Theta^{2}}{4}-2 \gamma-\beta_{0}-1\right)-(\gamma \Theta+\xi)\right] H(r)=0,
\end{aligned}
$$

which corresponds to the biconfluent Heun equation (BHE) $[88,107,108]$ and the function $H(r)$ is the biconflent Heun function :

$H(r)=H_{B}\left(2 \gamma, \Theta, \frac{\Theta^{2}}{4}-\beta_{0}, \xi ; r\right)$

Let us proceed with the search for bound states solutions, therefore we use the Frobenius method and the solution to equation (38) is a power series expansion (25) around the origin. We obtain the following recurrence relation :

$$
\begin{aligned}
c_{n+2}= & \frac{1}{(n+2)(n+2 \gamma+1)}\left[c_{n+1}\{\xi+\Theta(n+\gamma+1)\}\right. \\
& \left.+\left(2 n-\frac{\Theta^{2}}{4}+2 \gamma+\beta_{0}+1\right) c_{n}\right], \quad n \geq 1 .
\end{aligned}
$$

The coefficients of the power series expansion, for examples, $c_{1}$ and $c_{2}$ are 


$$
\begin{aligned}
c_{1}= & \left(\frac{\Theta}{2}+\frac{\xi}{2 \gamma}\right) c_{0}, \\
c_{2}= & \frac{1}{2(2 \gamma+1)}\left[c_{1}\{\xi+\Theta(\gamma+1)\}\right. \\
& \left.+\left(-\frac{\Theta^{2}}{4}+2 \gamma+\beta_{0}+1\right) c_{0}\right] .
\end{aligned}
$$

For the bound states solutions, we assume that the function $\psi(r)$ vanishes at $r \rightarrow 0$ and $r \rightarrow \infty$. Since we have written the function $H(r)$ as a power series expansion around the origin in Eq. (25), then, the relativistic bound state solutions can be achieved by imposing that the power series expansion (25) or the biconfluent Heun series becomes a polynomial of degree $n$. Through the recurrence relation (41), we can see that the power series expansion (25) becomes a polynomial of degree $n$ by imposing two conditions [31,89,107,109-111]:

$c_{n+1}=0, \quad\left(\frac{\Theta^{2}}{4}-2 \gamma-\beta_{0}-1\right)=2 n$,

where $n=1,2,3 \ldots$ Substituting various term into the Eq. (43) and analysing the energy quantization condition, we obtain the following eigenvalue equation:

$E_{n}^{2}-\left(2 n+2+\sqrt{1+4 k_{c}^{2}}\right) \alpha E_{n}-\left(p_{z}^{2}+m^{2}\right)=0$.

Therefore the relativistic energy eigenvalues of the system under a scalar potential of Coulomb-type are

$$
\begin{aligned}
E_{n}= & \left(n+1+\frac{1}{2} \sqrt{1+4 k_{c}^{2}}\right) \alpha \\
& +\sqrt{\left(n+1+\frac{1}{2} \sqrt{1+4 k_{c}^{2}}\right)^{2} \alpha^{2}+p_{z}^{2}+m^{2}} \\
= & 2\left(n+1+\frac{1}{2} \sqrt{1+4 k_{c}^{2}}\right) \Omega \\
& +\sqrt{4\left(n+1+\frac{1}{2} \sqrt{1+4 k_{c}^{2}}\right)^{2} \Omega^{2}+p_{z}^{2}+m^{2}} .
\end{aligned}
$$

And the corresponding eigenfunctions are

$\Psi_{n}(t, r, y, z)=C_{n} e^{-i\left(E_{n} t-p_{y} y-p_{z} z\right)} \psi_{n}(r)$,

where $\psi_{n}(r)$ is given by Eq. (38), and $C_{n}$ is the normalization constant. Thus the presence of Coulomb-type scalar potential, and the vorticity parameter modifies the levels of the system.

Note that the Eq. (45) does not represent the general expression for eigenvalue problem. One can obtain the eigenvalues one by one, that is, $E_{1}, E_{2}$ by imposing the additional condition $c_{n+1}=0$ in the recurrence relation. The solution with Heun's Equation makes it possible to obtain the eigenvalues one by one as done in Refs. $[109,110]$ and not explicited in general form by all eigenvalues $n$. To obtain the eigenvalues and corresponding eigenfunctions explicitly, two special cases corresponding to $n=1,2$ are study below in some details similar to that in $[109,110]$. The remaining cases corresponding to $n>2$ can be studied in the same way.

(1) For $n=1$, the conditions (43) are

$$
\left(\frac{\Theta^{2}}{4}-2 \gamma-\beta_{0}-1\right)=2 \quad, \quad c_{2}=0
$$

which implies

$c_{1}=\frac{2}{\{\xi+\Theta(\gamma+1)\}} c_{0}=\left(\frac{\Theta}{2}+\frac{\xi}{2 \gamma}\right) c_{0}$,

with the relation $\left(\frac{\Theta}{2}+\frac{\xi}{2 \gamma}\right)(\xi+\Theta(\gamma+1))=2$ and the corresponding eingenvalue and eigenfunction are

$$
\begin{aligned}
E_{1}= & 2\left(2+\sqrt{\frac{1}{4}+k_{c}^{2}}\right) \Omega \\
& +\sqrt{4\left(2+\sqrt{\frac{1}{4}+k_{c}^{2}}\right)^{2} \Omega^{2}+p_{z}^{2}+m^{2},} \\
\Psi_{1}= & C_{1} e^{-i\left(E_{1} t-p_{y} y-p_{z} z\right)} \psi_{1}(r), \\
\psi_{1}= & r^{\gamma} e^{-\frac{r}{2}(r+\Theta)}\left(c_{0}+c_{1} r\right) \\
= & r^{\gamma} e^{-\frac{r}{2}(r+\Theta)}\left[1+\frac{2}{\xi+\Theta(\gamma+1)} r\right] c_{0} .
\end{aligned}
$$

(2) For $n=2$, the conditions (43) are

$$
\left(\frac{\Theta^{2}}{4}-2 \gamma-\beta_{0}-1\right)=4 \quad, \quad c_{3}=0
$$

which implies

$$
\begin{aligned}
c_{1} & =\frac{2}{[\xi+\Theta(\gamma+1)]} c_{0}, \\
c_{2} & =\frac{2}{[\xi+\Theta(\gamma+2)]} c_{1} \\
& =\frac{4}{[\xi+\Theta(\gamma+2)][\xi+\Theta(\gamma+1)]} c_{0},
\end{aligned}
$$

and the corresponding eingenvalue and eigenfunction are

$$
\begin{aligned}
E_{2}= & 2\left(3+\sqrt{\frac{1}{4}+k_{c}^{2}}\right) \Omega \\
& +\sqrt{4\left(3+\sqrt{\frac{1}{4}+k_{c}^{2}}\right)^{2} \Omega^{2}+p_{z}^{2}+m^{2},} \\
\Psi_{2}= & C_{2} e^{-i\left(E_{2} t-p_{y} y-p_{z} z\right)} \psi_{2}(r),
\end{aligned}
$$




$$
\begin{aligned}
\psi_{2}= & r^{\gamma} e^{-\frac{r}{2}(r+\Theta)}\left(c_{0}+c_{1} r+c_{2} r^{2}\right) \\
= & r^{\gamma} e^{-\frac{r}{2}(r+\Theta)}[1 \\
& \left.+\frac{2}{(\xi+\Theta(\gamma+1))}\left\{1+\frac{2}{(\xi+\Theta(\gamma+2))} r\right\} r\right] c_{0} .
\end{aligned}
$$

Equation (36) can be transformed to the compact Liouville's normal form

$$
\left(\frac{d^{2}}{d r^{2}}-\sum_{i=-2}^{2} a_{i} r^{i}\right) \psi(r)=0
$$

where the coefficients are given by

$a_{-2}=k^{2}, \quad a_{-1}=\xi, \quad a_{0}=\beta_{0}, \quad a_{1}=\Theta, \quad a_{2}=1$.

In this stage, to solve Eq. (53), one can use the generalized series method by Eshghi et al. [99, 112,113], and the parameters $a_{0}, a_{1}, a_{2}, a_{-1}$ and $a_{-2}$ are given in Eq. (54), and by a little algebraic calculation, one can evaluate eigenvalues of the problem.

\subsection{Spin-0 particle with vector and scalar potentials of}

Coulomb-type in flat Gödel-type metric

Here we study solutions of the Klein-Gordon equation with vector and scalar potentials of Coulomb-type.

We have considered the Coulomb-type vector potential $A_{0}=V=\frac{\kappa_{0}}{x}$ and the Coulomb-type scalar potential $S=\frac{k_{c}}{x}$, where $\kappa_{0}$ and $k_{c}$ are constants. Using the space-time (9), we obtain the following equation from (8)

$$
\begin{aligned}
\partial_{\mu} & \left(g^{\mu \nu} \partial_{\nu} \Psi\right)-i e\left[\partial_{\mu}\left(g^{\mu \nu} A_{\nu} \Psi\right)+A_{\mu} g^{\mu \nu} \partial_{\nu} \Psi\right] \\
& -e^{2} g^{\mu \nu} A_{\mu} A_{\nu} \Psi=(m+S)^{2} \Psi \\
\Rightarrow & {\left[-\partial_{t}^{2}+\left(\alpha x \partial_{t}-\partial_{y}\right)^{2}+\partial_{x}^{2}+\partial_{z}^{2}\right.} \\
& \left.+2 i e\left\{\frac{\kappa_{0}}{x}\left(1-\alpha^{2} x^{2}\right) \partial_{t}+\alpha \kappa_{0} \partial_{y}\right\}\right] \Psi \\
& +\kappa_{0}^{2} e^{2}\left(\frac{1}{x^{2}}-\alpha^{2}\right) \Psi=\left(m+\frac{k_{c}}{x}\right)^{2} \Psi .
\end{aligned}
$$

The equation (55) is independent of $t, y, z$, so it is appropriate to choose the ansatz given in (16), we obtain the following differential equation :

$$
\begin{aligned}
& \psi^{\prime \prime}(x)-\left(\alpha^{2} E^{2} x^{2}+2 \alpha E p_{y} x+\frac{k_{c}^{2}}{x^{2}}+\frac{2 m k_{c}}{x}\right) \psi(x) \\
& +2 e\left[\frac{\kappa_{0} E}{x}\left(1-\alpha^{2} x^{2}\right)-\alpha \kappa_{0} p_{y}\right] \psi \\
& +\kappa_{0}^{2} e^{2}\left(\frac{1}{x^{2}}-\alpha^{2}\right) \psi=\beta \psi(x)
\end{aligned}
$$

$$
\begin{aligned}
\Rightarrow & \psi^{\prime \prime}(x)-\left[\alpha^{2} E^{2} x^{2}+2 \alpha E\left(p_{y}+\alpha e \kappa_{0}\right) x\right. \\
& \left.+\frac{k_{c}^{2}-e^{2} \kappa_{0}^{2}}{x^{2}}+\frac{2\left(m k_{c}-e \kappa_{0} E\right)}{x}\right] \psi \\
= & \left(\beta+\kappa_{0}^{2} e^{2} \alpha^{2}+2 e \alpha \kappa_{0} p_{y}\right) \psi(x) .
\end{aligned}
$$

Let us define $r=\sqrt{\alpha E} x$, then the Eq. (56) becomes

$\psi^{\prime \prime}(r)-\left[r^{2}+\frac{L^{2}}{r^{2}}+\Phi r+\frac{\zeta}{r}\right] \psi(r)=\gamma_{0} \psi(r)$,

where

$\Phi=\frac{2\left(p_{y}+\alpha e \kappa_{0}\right)}{\sqrt{\alpha E}}, \quad L=\sqrt{k_{c}^{2}-e^{2} \kappa_{0}^{2}}$,

$\zeta=\frac{2\left(m k_{c}-e \kappa_{0} E\right)}{\sqrt{\alpha E}}$

$\gamma_{0}=\frac{\left(\beta+\kappa_{0}^{2} e^{2} \alpha^{2}+2 e \alpha \kappa_{0} p_{y}\right)}{\alpha E}$.

The solution to Eq. (57) is

$\psi(r)=r^{\sigma} e^{-\frac{r^{2}}{2}} e^{-\frac{\Phi r}{2}} H(r), \quad \sigma=\frac{1}{2}\left[1+\sqrt{1+4 L^{2}}\right]$,

where we have assumed $k_{c}^{2}>e^{2} \kappa_{0}^{2}$. Substituting Eq. (59) into Eq. (57), we obtain

$$
\begin{aligned}
& r H^{\prime \prime}(r)+\left[2 \sigma-r \Phi-2 r^{2}\right] H^{\prime}(r) \\
& \quad+\left[r\left(\frac{\Phi^{2}}{4}-2 \sigma-\gamma_{0}-1\right)-(\sigma \Phi+\zeta)\right] H(r)=0,
\end{aligned}
$$

the biconfluent Heun equation (BHE) $[88,107,108]$ and the function $H(r)$ is the biconflent Heun function :

$H(r)=H_{B}\left(2 \sigma, \Phi, \frac{\Phi^{2}}{4}-\gamma_{0}, \zeta ; r\right)$

The solution of Eq. (61) is a power series expansion (25) around the origin. We obtain the following recurrence relation :

$$
\begin{aligned}
c_{n+2}= & \frac{1}{(n+2)(n+2 \sigma+1)}\left[c_{n+1}\{\zeta+\Phi(n+\sigma+1)\}\right. \\
& \left.+\left(2 n-\frac{\Phi^{2}}{4}+2 \sigma+\gamma_{0}+1\right) c_{n}\right], \quad n \geq 1 .
\end{aligned}
$$


The coefficients of the power series expansion, for examples, $c_{1}$ and $c_{2}$ are

$$
\begin{aligned}
c_{1}= & \left(\frac{\Phi}{2}+\frac{\zeta}{2 \sigma}\right) c_{0}, \\
c_{2}= & \frac{1}{2(2 \sigma+1)}\left[c_{1}\{\zeta+\Phi(\sigma+1)\}\right. \\
& \left.+\left(-\frac{\Phi^{2}}{4}+2 \sigma+\gamma_{0}+1\right) c_{0}\right] .
\end{aligned}
$$

The power series expansion becomes a polynomial of $r^{n}$ by imposing the following two condition:

$c_{n+1}=0, \quad\left(2 \sigma+\gamma_{0}-\frac{\Phi^{2}}{4}+1\right)=-2 n$.

Substituting various terms into Eq. (64) and analysing the condition, we get the energy eigenvalues equation:

$E_{n}^{2}-\left(2 n+2+\sqrt{1+4 L^{2}}\right) \alpha E_{n}-\left(p_{z}^{2}+m^{2}\right)=0$.

Therefore the relativistic energy eigenvalues of the system are

$$
\begin{aligned}
E_{n}= & \left(n+1+\frac{1}{2} \sqrt{1+4 L^{2}}\right) \alpha \\
& +\sqrt{\left(n+1+\frac{1}{2} \sqrt{1+4 L^{2}}\right)^{2} \alpha^{2}+p_{z}^{2}+m^{2}} \\
= & 2 \Omega\left(n+1+\frac{1}{2} \sqrt{1+4 L^{2}}\right) \\
& +\sqrt{4 \Omega^{2}\left(n+1+\frac{1}{2} \sqrt{1+4 L^{2}}\right)^{2}+p_{z}^{2}+m^{2}},
\end{aligned}
$$

where $L=\sqrt{k_{c}^{2}-e^{2} \kappa_{0}^{2}}$. Thus the presence of scalar and vector potentials of Coulomb-type, and the vorticity parameter modifies the levels. The corresponding eigenfunctions are

$\Psi_{n}=B_{n} e^{-i\left(E_{n} t-p_{y} y-p_{z} z\right)} \psi_{n}(r)$,

where $\psi_{n}(r)$ is given by Eq. (59), and $B_{n}$ is the normalization constant. Note that if one takes $\kappa_{0}=0$, that is, without any vector potential of Coulomb-type, we get back Eq. (45) the energy eignevalues of the system with only scalar potential of Coulomb-type. On the other hand, for $k_{c}=0$, that is, no scalar potential of Coulomb-type, we get the following energy eigenvalues of spin- 0 particles under a vector potential of Coulomb-type

$$
\begin{aligned}
E_{n}= & 2 \Omega\left(n+1+\sqrt{\frac{1}{4}-e^{2} \kappa_{0}^{2}}\right) \\
& +\sqrt{4 \Omega^{2}\left(n+1+\sqrt{\frac{1}{4}-e^{2} \kappa_{0}^{2}}\right)^{2}+p_{z}^{2}+m^{2}} .
\end{aligned}
$$

Here also the Eq. (66) does not represent the general expression for eigenvalue problem. One can obtain the eigenvalues one by one, that is, $E_{1}, E_{2}$ by imposing the additional condition $c_{n+1}=0$ in the recurrence relation. The solution with Heun's Equation makes it possible to obtain the eigenvalues one by one and but not explicited in general form by all eigenvalues $n$. The eigenfunctions and corresponding eigenvalues for two special cases corresponding to $n=1,2$ are studied below in some details (see Refs. $[109,110]$ ). The remaining cases corresponding to $n>2$ can be studied in the same way.

(1) For $n=1$, the conditions (64) are

$\left(\frac{\Phi^{2}}{4}-2 \sigma-\gamma_{0}-1\right)=2 \quad, \quad c_{2}=0$

which implies

$c_{1}=\frac{2}{\{\zeta+\Phi(\sigma+1)\}} c_{0}=\left(\frac{\Phi}{2}+\frac{\zeta}{2 \sigma}\right) c_{0}$,

with the relation $\left(\frac{\Phi}{2}+\frac{\zeta}{2 \sigma}\right)(\zeta+\Phi(\sigma+1))=2$ and the corresponding eingenvalues and eigenfunctions from (66) and (67) are

$$
\begin{aligned}
E_{1}= & 2\left(2+\sqrt{\frac{1}{4}+k_{c}^{2}-e^{2} \kappa_{0}^{2}}\right) \Omega \\
& +\sqrt{4\left(2+\sqrt{\frac{1}{4}+k_{c}^{2}-e^{2} \kappa_{0}^{2}}\right)^{2} \Omega^{2}+p_{z}^{2}+m^{2}} \\
\Psi_{1}= & B_{1} e^{-i\left(E_{1} t-p_{y} y-p_{z} z\right)} \psi_{1}(r), \\
\psi_{1}= & r^{\sigma} e^{-\frac{r}{2}(r+\Phi)}\left(c_{0}+c_{1} r\right) \\
= & r^{\sigma} e^{-\frac{r}{2}(r+\Phi)}\left[1+\frac{2}{\zeta+\Phi(\sigma+1)} r\right] c_{0} .
\end{aligned}
$$

(2) For $n=2$, the conditions (64) are

$$
\left(\frac{\Phi^{2}}{4}-2 \sigma-\gamma_{0}-1\right)=4, \quad c_{3}=0
$$


which implies

$$
\begin{aligned}
c_{1} & =\frac{2}{\{\zeta+\Phi(\sigma+1)\}} c_{0}, \\
c_{2} & =\frac{2}{\{\zeta+\Phi(\sigma+2)\}} c_{1} \\
& =\frac{4 c_{0}}{[\zeta+\Phi(\sigma+2)][\zeta+\Phi(\sigma+1)]},
\end{aligned}
$$

and the corresponding eingenvalue and eigenfunction are

$$
\begin{aligned}
E_{2}= & 2\left(3+\sqrt{\frac{1}{4}+k_{c}^{2}-e^{2} \kappa_{0}^{2}}\right) \Omega \\
& +\sqrt{4\left(3+\sqrt{\frac{1}{4}+k_{c}^{2}-e^{2} \kappa_{0}^{2}}\right)^{2} \Omega^{2}+p_{z}^{2}+m^{2},} \\
\Psi_{2}= & B_{2} e^{-i\left(E_{2} t-p_{y} y-p_{z} z\right)} \psi_{2}(r), \\
\psi_{2}= & r^{\sigma} e^{-\frac{r}{2}(r+\Phi)}\left(c_{0}+c_{1} r+c_{2} r^{2}\right) \\
= & r^{\sigma} e^{-\frac{r}{2}(r+\Phi)}[1 \\
& \left.+\frac{2}{\zeta+\Phi(\sigma+1)}\left\{1+\frac{2}{\zeta+\Phi(\sigma+2)} r\right\} r\right] c_{0} .
\end{aligned}
$$

Eq. (57) can be transformed to the compact Liouville's normal form

$$
\left(\frac{d^{2}}{d r^{2}}-\sum_{i=-2}^{2} a_{i} r^{i}\right) \psi(r)=0
$$

where the coefficients are

$a_{-2}=L^{2}, \quad a_{-1}=\zeta, \quad a_{0}=\gamma, \quad a_{1}=\Phi, \quad a_{2}=1$.

To solve Eq. (75), one can use the generalized series method by Eshghi et al. [99,112,113], and by a little algebraic calculation, one can evaluate eigenvalues of the problem.

\section{Results}

Before presenting our result, let us present the recent studies $[28,58,77,78,107,114]$ on the problems involving Linear, Coulomb-type and other interactions on the Klein-Gordon equation and the Heun's differential equation.

In Ref. [77], the second order Heun differential equation is of the form

$$
H^{\prime \prime}(r)+\left[\frac{2|l|+1}{r}-\delta-2 r\right] H^{\prime}(r)+\left[g-\frac{h}{r}\right] H(r)=0 .
$$

The energy eigenvalues are

$$
\begin{aligned}
E_{n, l}^{2}= & m^{2}+2 m \sqrt{\omega_{n, l}^{2}+\frac{\mu^{2}}{m^{2}}}(n+|l|+1)-m \omega_{n, l} \\
& -\frac{\mu^{2}}{\left(\omega_{n, l}^{2}+\frac{\mu^{2}}{m^{2}}\right)},
\end{aligned}
$$

Equation (78) corresponds to the spectrum for spinless particle in the Klein-Gordon oscillator subject to a linear confining potential in $(2+1)$-dimension.

In Ref. [78], the second order Heun differential equation is of the form

$$
\begin{aligned}
& H^{\prime \prime}(r)+\left[\frac{2|\gamma|+1}{r}-2 r\right] H^{\prime}(r) \\
& +\left[\frac{\beta}{m \omega}-2-2 \gamma+\frac{\delta}{r}\right] H(r)=0 .
\end{aligned}
$$

The energy eigenvalues are

$E_{n, l}^{2}=m^{2}+m \omega_{n, l}(2 n+2|\gamma|+1)$.

Equation (80) corresponds to the spectrum of energy of the Klein-Gordon oscillator under the influence of a Coulomb potential.

In addition, the Klein-Gordon oscillator under the influence of a Coulomb potential and linear scalar potential in Ref. [78], the second order Heun differential equation is of the form

$$
H^{\prime \prime}(\xi)+\left[\frac{2|\gamma|+1}{\xi}-\mu-2 \xi\right] H^{\prime}(\xi)+\left[\sigma+\frac{\varphi}{\xi}\right] H(\xi)=0
$$

The energy eigenvalues are

$$
\begin{aligned}
& E_{n, l}^{2}=m^{2}-m \omega_{n, l}+2 \Theta_{n, l}(n+|\gamma|+1)-\frac{m^{2} \varphi^{2}}{\Theta_{n, l}^{2}} \\
& \Theta_{n, l}=\sqrt{m^{2} \omega_{n, l}^{2}+\varphi^{2}}
\end{aligned}
$$

Equation (82) corresponds to the spectrum of energy of the Klein-Gordon oscillator under the influence of a Coulomb potential and the linear scalar potential.

In Ref. [107], the second order Heun differential equation is of the form

$$
H^{\prime \prime}(\xi)+\left[\frac{2|l|+1}{\xi}-2 \xi\right] H^{\prime}(\xi)+\left[g+\frac{d}{\xi}\right] H(\xi)=0 .
$$

The energy eigenvalues are

$$
E_{n, l}=\omega(n+|l|+1)+\frac{Q^{2} \lambda_{m}^{2}}{8 m}+\frac{k^{2}}{2 m} .
$$


Equation (84) is the energy levels of the two-dimensional harmonic oscillator under the influence of Coulomb-type potential induced by the interaction between the electric quadrupole moment and magnetic field.

In Ref. [58], the second order Heun differential equation is of the form

$$
H^{\prime \prime}(\xi)+\left[\frac{\Theta}{\xi}-\alpha-2 \xi\right] H^{\prime}(\xi)+\left[g-\frac{\alpha \Theta+2 \delta}{2 \xi}\right] H(\xi)=0 .
$$

The energy eigenvalues are

$$
E_{n, l}=\omega(n+|l|+1)-\frac{\eta^{2}}{2 m \omega^{2}}+\frac{\mu^{2} \lambda^{2}}{8 m}+\frac{k^{2}}{2 m} .
$$

Equation (86) corresponds to the spectrum of energy of a moving atom with a magnetic quadrupole moment subject to a harmonic potential and a linear confining potential under the influence of an analogue of the Coulomb potential.

In Ref. [114], the second order Heun differential equation is of the form

$$
H^{\prime \prime}(r)+\left[\frac{2|\gamma|+1}{r}-2 r\right] H^{\prime}(r)+\left[v+\frac{v}{r}\right] H(r)=0 .
$$

The energy eigenvalues are

$E_{n, l}=\sqrt{\frac{\omega_{n, l}^{2}}{4}+\Omega \omega_{n, l}}(n+|\gamma|+1)-\frac{1}{2} l \omega_{n, l}-\Omega l$.

Equation (88) corresponds to the energy levels obtained from the effects of the Kratzer potential and rotating effects on the Landau-type system for a neutral particle (atom, molecule) with an induced electric dipole moment.

In Ref. [28], the linear confinement of a scalar particle in Som-Raychaudhuri space-time with a cosmic string, the second order Heun differential equation is of the form

$$
\begin{aligned}
& H^{\prime \prime}(x)+\left[\frac{\frac{2|\gamma|}{\alpha}+1}{x}-\theta-2 x\right] H^{\prime}(x) \\
& +\left[\beta+\frac{\theta^{2}}{4}-2-2|l|-\frac{\theta\left(\frac{2|\gamma|}{\alpha}+1\right)}{2 x}\right] H(x)=0 .
\end{aligned}
$$

The energy eigenvalues equation is

$$
\begin{aligned}
& E_{n, l}^{2}-\frac{2 \Omega l}{\alpha} E_{n, l}-C_{n, l}=0, \\
& C_{n, l}=M^{2}+p_{z}^{2}+2 \omega_{n, l}\left(n+\frac{|l|}{\alpha}+1\right)-\frac{k^{2} M^{2}}{\omega_{n, l}^{2}} .
\end{aligned}
$$

Eq. (90) corresponds to the energy levels of polynomial of degree $n$ of a scalar particle in the Klein-Gordon equation under the influence of linear scalar potential with a cosmic string.

In the present work, the confinement of a scalar particle in a topologically trivial flat Gödel-type space-time with a linear scalar potential, the eigenvalues and eigenfunctions corresponding to $n=1,2$ are

$$
\begin{aligned}
n=1: \quad E_{1}= & 3 \Omega+\sqrt{9 \Omega^{2}+m^{2}+p_{z}^{2}+A_{1}}, \\
A_{1}= & \frac{3}{2}\left(1+\frac{k_{L}^{2}}{2 \omega^{2}}\right) \frac{k_{L}^{2}}{\omega}+\left(p_{y}^{2}-m^{2}\right) \frac{k_{L}^{2}}{\omega^{2}} \\
& -2 m k_{L} p_{y}\left(1-\frac{k_{L}^{2}}{2 \omega^{2}}\right), \\
\psi_{1}(r)= & e^{-\frac{r}{2}\left(r+\delta_{0}\right)}\left(1+\frac{2}{\delta_{0}} r\right) c_{0}, \\
n=2: \quad E_{2}= & 5 \Omega+\sqrt{25 \Omega^{2}+m^{2}+p_{z}^{2}+A_{2}}, \\
A_{2}= & \frac{5}{2}\left(1+\frac{k_{L}^{2}}{2 \omega^{2}}\right) \frac{k_{L}^{2}}{\omega}+\left(p_{y}^{2}-m^{2}\right) \frac{k_{L}^{2}}{\omega^{2}} \\
& -2 m k_{L} p_{y}\left(1-\frac{k_{L}^{2}}{2 \omega^{2}}\right), \\
\psi_{2}(r)= & e^{-\frac{r}{2}\left(r+\delta_{0}\right)}\left[1+\frac{4\left(\delta_{0}+r\right)}{\delta_{0}^{2}-2} r\right] c_{0} .
\end{aligned}
$$

The confinement of a scalar particle in a flat Gödel-type space-time under Coulomb-type scalar potential, the second order Heun differential equation is of the form

$$
\begin{aligned}
& r H^{\prime \prime}(r)+\left[2 \gamma-\Theta r-2 r^{2}\right] H^{\prime}(r) \\
& \quad+\left[r\left(\frac{\Theta^{2}}{4}-2 \gamma-\beta_{0}-1\right)-(\gamma \Theta+\xi)\right] H(r)=0 .
\end{aligned}
$$

The relativistic energy eigenvalues for $n^{\text {th }}$ degree polynomial are

$$
\begin{aligned}
E_{n}= & 2 \Omega\left(n+1+\sqrt{\frac{1}{4}+k_{L}^{2}}\right) \\
& +\sqrt{4 \Omega^{2}\left(n+1+\sqrt{\frac{1}{4}+k_{L}^{2}}\right)^{2}+p_{z}^{2}+m^{2}} .
\end{aligned}
$$

The relativistic energy eigenvalues and eigenfunctions corresponding to $n=1,2$ has given in explicitly in Eq. (49), Eq. (52) and others are in the same way.

The Confinement of spin-0 particles in flat Gödel-type space-time under a vector and scalar potential of Coulombtype, the second order Heun differential equation is of the form 


$$
\begin{aligned}
& r H^{\prime \prime}(r)+\left[2 \sigma-r \Phi-2 r^{2}\right] H^{\prime}(r) \\
& \quad+\left[r\left(\frac{\Phi^{2}}{4}-2 \sigma-\gamma_{0}-1\right)-(\sigma \Phi+\zeta)\right] H(r)=0 .
\end{aligned}
$$

The relativistic energy eigenvalues for $n^{\text {th }}$ degree polynomial are

$$
\begin{aligned}
E_{n}= & 2 \Omega\left(n+1+\sqrt{\frac{1}{4}+k_{c}^{2}-e^{2} \kappa_{0}^{2}}\right) \\
& +\sqrt{4 \Omega^{2}\left(n+1+\sqrt{\frac{1}{4}+k_{c}^{2}-e^{2} \kappa_{0}^{2}}\right)^{2}+p_{z}^{2}+m^{2}} .
\end{aligned}
$$

The relativistic energy eigenvalues and eigenfunctions corresponding to $n=1,2$ has given more explicitly in Eqs. (71), (74) and others are in the same way.

Note that for solutions investigated in this article, we do not have a general expression for the eigenvalues of energy. For solutions that have discussed in sub-section 3.1 and subsection 3.2, the Eqs. (45) and (66) are not represent the general expression for eigenvalues of energy. By imposing the additional condition $c_{n+1}=0$ in the recurrence relation, one can get solutions for each level individually as done in Refs. $[109,110]$. We have evaluated these levels corresponding to $n=1,2$ by Eq. (49), Eq. (52) and Eq. (71), Eq. (74) for examples. Similarly, the Eq. (91), Eq. (92) are the eigenvalues for each level due to the imposition of the additional condition $c_{n+1}=0$.

\section{Conclusions}

The relativistic energy levels of a scalar and spin-0 particles with linear scalar potential in Gödel universe, Gödeltype solutions in flat, spherical and hyperbolic spaces with or without cosmic string, were investigated by several authors. In Ref. [28], authors have shown that the vorticity and the topological defect stem from a particular Gödel-type solution called the Som-Raychaudhuri space-time with a cosmic string. By analysing the energy associated with the radial mode, they have shown that both the vorticity and the topology of the cosmic string modifies the energy eigenvalues and give rise to the allowed energies. Note that the line element considered for the Som-Raychaudhuri space-time in [28] is for the cylindrical symmetry system and by solving the Klein-Gordon equation they obtained the energy eigenvalues. In Ref. [99], authors considered the cylindrical symmetry Som-Raychaudhuri space-time in the presence of a topological defect, and analyze the Yukawa-like confinement of a relativistic scalar particle. They have calculated the energy eigenvalues and wave-functions by using the generalized series method. In Ref. [94], spin-0 equation in the presence of a vector and a scalar potential, were investigated. They examined the wave equation on the Klein-Gordon oscillator in a cosmic string space-time and have shown that the potentials allow the formation of bound states, and the topological defect modifies the energy levels of the system. In Ref. [23], the obtained energy eigenvalues of flat Gödel-type spacetime (Som-Raychaudhuri metric) is reduced to the energy levels obtained in $[21,30]$ and to the Landau levels in the presence of cosmic string [31].

We have analysed the influence of a linear scalar potential on the Klein-Gordon equation in a topologically trivial flat Gödel-type space-time. The flat Gödel-type space-time considered here is in cartesian coordinates system and we have solved the Klein-Gordon equation in this spac-time with a linear scalar potential. We have seen that Eq. (31) the energy eigenvalues of the system get modified by the presence of the confining potential, and the vorticity parameter $\Omega$ characterising the space-time. In the energy eigenvalues, the ground state is determined by the quantum number $n=1$ instead of the quantum number $n=0$. Observe that the present result is different from the one obtained in Ref. [28] because of the cylindrical symmetry Som-Raychaudhuri space-time whereas, the present Gödel-type flat solution is in the cartesian coordinates system. Similarly, the obtained result is different from the one presented in Ref. [99]. Models with confining potentials have been used to describe the spectrum of quarkonia-type systems. In the case of linear confining potential, this would be the confining potential of quarks. This type of potential has been used to investigate quarkonia in heavy quark-model. Thereby, the study of the linear confinement of relativistic scalar particle in the studied space-time can be used as Quarkonia-type systems to investigate heavy quarkonia where, the rotation plays the role of external uniform magnetic field. We have also considered the Coulomb-type scalar potential on the Klein-Gordon equation and seen that the presence of Coulomb-like scalar potential modifies the energy levels and Eq. (45) the $n^{\text {th }}$ degree polynomial of energy spectrum associated with this equation depends on the confining scalar potential, and the vorticity parameter and Eq. (46) the corresponding eigenfunctions. We have evaluated the eigenvalues and eigenfunctions for the special cases corresponding to $n=1,2$ more explicitly Eqs. (49), (52). Furthermore, we have obtained Eq. (66) the energy eigenvalues for $n^{\text {th }}$ degree polynomial with vector and scalar potentials of Coulomb-type by solving the relativistic Klein-Gordon equation of spin-0 particles. We have seen that the energy eigenvalues of the system get modifies and depend on the vorticity parameter, and the vector $\left(\kappa_{0}\right)$ and scalar potential $\left(k_{c}\right)$ of Coulomb-type. We have evaluated the energy eigenvalues and eigenfunction for the special cases corresponding to $n=1,2$ in more details Eq. (71), Eq. (74) more explicitly. Note that the Eq. (45) and Eq. (66) are 
not represent the general expression for eigenvalues problem. One can obtain the eigenvalues one by one, that is, $E_{1}, E_{2}$ by imposing the condition $c_{n+1}=0$ in the recurrence relation. The solution with Heun's Equation makes it possible to obtain the eigenvalues one by one as done in Refs. [109,110] and not explicited in general form by all eigenvalues $n$. We have evaluated solution for each level individually corresponding to $n=1,2$ by Eq. (49), Eq. (52), Eq. (71), Eq. (74) and Eq. (91), Eq. (92) for examples.

So, in this paper, we have shown some results about quantum systems where general relativistic effects are taken into account, that in addition with the previous results $[21,22,28,35,94,98,99]$ the present may possess some interesting effects.

Acknowledgements I would like to thank the annoymous kind referee(s) for the positive suggestions and valuable comments which have greatly improved the present text. I am also very much thankful to the Academic Editor for giving the opportunity for revising this work.

Data Availability Statement This manuscript has no associated data or the data will not be deposited. [Authors' comment: We have not use any data for this manuscript or there are no data associated with this manuscript.]

Open Access This article is distributed under the terms of the Creative Commons Attribution 4.0 International License (http://creativecomm ons.org/licenses/by/4.0/), which permits unrestricted use, distribution, and reproduction in any medium, provided you give appropriate credit to the original author(s) and the source, provide a link to the Creative Commons license, and indicate if changes were made. Funded by SCOAP ${ }^{3}$.

\section{References}

1. F. Ahmed, Eur. Phys. J. C 78(7), 598 (2018)

2. K. Gödel, Rev. Mod. Phys. 21, 447 (1949)

3. F. Ahmed, Eur. Phys. J. C 78(5), 385 (2018)

4. F. Ahmed, J. Phys. Commun. 2(3), 035036 (2018)

5. S.W. Hawking, Phys. Rev. D 46, 603 (1992)

6. M.S. Morris, K.S. Thorne, U. Yurtsever, Phys. Rev. Lett. 61, 1446 (1988)

7. M.S. Morris, K.S. Thorne, Am. J. Phys. 56, 395 (1988)

8. A. Ori, Phys. Rev. Lett. 71, 2517 (1993)

9. A. Ori, Y. Soen, Phys. Rev. D 49, 3990 (1994)

10. Y. Soen, A. Ori, Phys. Rev. D 54, 4858 (1996)

11. M.J. Reboucas, J. Tiomno, Phys. Rev. D 28, 1251 (1983)

12. M. Reboucas, J. Aman, A.F.F. Teixeira, J. Math. Phys. 27, 1370 (1985)

13. M.O. Galvao, M. Reboucas, A.F.F. Teixeira, W.M. Silva, J. Math. Phys. 29, 1127 (1988)

14. M. Rooman, P. Spindel, Class. Quantum Gravity 15, 3241 (1998)

15. M.M. Som, A.K. Raychaudhuri, Proc. R. Soc. A 304, 81 (1968)

16. G.T. Horowitz, A.A. Tseytlin, Phys. Rev. D 51, 2896 (1995)

17. J.G. Russo, A.A. Tseytlin, Nucl. Phys. B 448, 293 (1995)

18. J.G. Russo, A.A. Tseytlin, Nucl. Phys. B 454, 164 (1995)

19. E.K. Boyda, S. Ganguli, P. Horava, U. Varadarajan, Phys. Rev. D 67, 106003 (2003)

20. T. Harmark, T. Takayanagi, Nucl. Phys. B 662, 3 (2003)
21. N. Drukker, B. Fiol, J. Simon, JCAP 0410, 012 (2004)

22. S. Das, J. Gegenberg, Gen. Rel. Grav. 40, 2115 (2008)

23. J. Carvalho, A. M. de M. Carvalho, C. Furtado, Eur. Phys. J. C 74, 2935 (2014)

24. J. Carvalho, A. M. de M. Carvalho, E. Cavalcante, C. Furtado, Eur. Phys. J. C 76, 365 (2016)

25. G. Q. Garcia, J. R. de S. Oliveira, K. Bakke, C. Furtado, EPJ Plus 132, 123 (2017)

26. F.M. Paiva, M.J. Reboucas, A.F.F. Teixeira, Phys. Lett. A 126(3), 168 (1987)

27. Z. Wang, Z. Long, C. Long, M. Wu, EPJ Plus 130, 36 (2015)

28. R.L.L. Vitoria, C. Furtado, K. Bakke, Eur. Phys. J. C 78, 44 (2018)

29. B.D. Figueiredo, I.D. Soares, J. Tiomno, Class. Quantum Grav. 9, 1593 (1992)

30. N. Drukker, B. Fiol, J. Simon, Phys. Rev. Lett. 91, 231601 (2003)

31. C. Furtado, B.G.C. da Cunha, F. Moraes, E.R.B. de Mello, V.B. Bezerra, Phys. Lett. A 195(1), 90 (1994)

32. G. Q. Garcia, J. R. de S. Oliveira, C. Furtado, Int. J. Mod. Phys. D 27, 1850027 (2018)

33. S. G. Fernandes, G. de A. Marques, V. B. Bezerra, Class. Quantum Grav. 23, 7063 (2006)

34. A. Havare, T. Yetkin, Class. Quantum Grav. 19, 1 (2002)

35. L.C.N. Santos, C.C. Barros Jr., Eur. Phys. J. C 76, 560 (2016)

36. A. de Souza Dutra, C.-S. Jia, Phys. Lett. A 352, 484 (2006)

37. W.-C. Qiang, R.-S. Zhou, Y. Gao, Phys. Lett. A 371, 201 (2007)

38. A.S. de Castro, Phys. Lett. A 338, 81 (2005)

39. A.D. Alhaidari, H. Bahlouli, A. Al-Hasan, Phys. Lett. A 349, 87 (2006)

40. F. Domingues-Adame, Phys. Lett. A 136, 175 (1989)

41. Y. Xu, S. He, C.-S. Jia, Phys. Scr. 81, 045001 (2010)

42. A. de Souza Dutra, G. Chen, Phys. Lett. A 349, 297 (2006)

43. G. Chen, Phys. Lett. A 339, 300 (2005)

44. F. Dominguez-Adame, Phys. Lett. A 136, 175 (1989)

45. S.M. Ikhdair, Eur. Phys. J. A 40, 143 (2009)

46. Z.Q. Ma, S.H. Dong, X.Y. Gu, J. Yu, Int. J. Mod. Phys. E 13, 597 (2004)

47. A.D. Alhaidari, Phys. Lett. A 322, 12 (2004)

48. C.S. Jia, J.-Y. Liu, P.-Q. Wang, C.S. Che, Phys. Lett. A 369, 274 (2007)

49. C.S. Jia, A.S. Dutra, Ann. Phys. 323, 566 (2008)

50. O. Mustafa, S.H. Mazharimousavi, Int. J. Theor. Phys. 47, 446 (2008)

51. C.L. Chrichfield, J. Math. Phys. 17, 261 (1976)

52. E.J. Austin, Mol. Phys. 40, 893 (1980)

53. E.R. Vrscay, Phys. Rev. A 31, 2054 (1985)

54. K. Killingbeck, Rep. Prog. Phys. 40, 963 (1977)

55. K. Killingbeck, Phys. Lett. A 65, 87 (1978)

56. R.P. Saxena, V.S. Varma, J. Phys. A : Math. Gen. 15, L149 (1982)

57. E. Castro, P. Martin, J. Phys. A : Math. Gen. 33, 5321 (2000)

58. I.C. Fonseca, K. Bakke, Proc. R. Soc. A 471, 20150362 (2015). arXiv:1603.03078 [quant-ph]

59. G. Plante, A.F. Antippa, J. Math. Phys. 46, 062108 (2005)

60. J.H. Noble, U.D. Jentschura, Int. J. Mod. Phys. A 30, 1550002 (2015)

61. M.L. Glasser, N. Shawagfeh, J. Math. Phys. 25, 2533 (1984)

62. H. Tezuka, AIP Adv. 3, 082135 (2013)

63. J.F. Gunion, L.F. Li, Phys. Rev. D 12, 3583 (1975)

64. P. Ferreira Leal, Phys. Rev. D 38, 2648 (1988)

65. F. Dominguez-Adame, M.A. Gonzalez, Euro phys. Lett. 13, 193 (1990)

66. J.Y. Guo, J.C. Han, R.D. Wang, Phys. Lett. A 353, 378 (2006)

67. F.L. Lu, C.Y. Chen, Chin. Phys. B 19, 100309 (2010)

68. A.S. Dutra, G. Chen, Phys. Lett. A 349, 297 (2006)

69. K.J. Oyewumi, C.O. Akoshile, Euro. Phys. J. A. 45, 311 (2010)

70. E. Olgar, R. Koc, H. Tutunculer, Chin. Phys. Lett. 23, 539 (2006)

71. S.M. Ikhdair, R. Sever, J. Math. Phys. 52, 122108 (2011) 
72. S.M. Ikhdair, R. Sever, J. Phys. A : Math. Theor. 44, 355301 (2011)

73. C.A. Onate, M.C. Onyeaju, A.N. Ikot, J.O. Ojonubah, Chin. J. Phys. 54, 820 (2016)

74. O.J. Oluwadare, K.J. Oyewumi, C.O. Akoshile, O.A. Babalola, Phys. Scr. 86, 035002 (2012)

75. H. Hassanabadi, S. Zarrinkamar, H. Rahimov, Commun. Theor. Phys. 53, 423 (2011)

76. H. Hassanabadi, H. Rahimov, S. Zarrinkamar, Annalen der Physik 523, 566 (2011)

77. R.L.L. Vitoria, K. Bakke, EPJ Plus 131, 36 (2016)

78. R. L. L. Vitoria, C. Furtado, K. Bakke, Ann. Phys. (N. Y.) 370, 128 (2016). arXiv:1511.05072 [quant-ph]

79. R.L.L. Vitoria, K. Bakke, Gen. Rel. Grav. 48, 161 (2016)

80. Q. Wen-Chao, Chin. Phys. 12, 1054 (2003)

81. H. Motavalli, A.R. Akbarieh, Mod. Phys. Lett. A 25, 2523 (2010)

82. F. Yasuk, A. Durmus, I. Boztosun, J. Math. Phys. 47, 082302 (2006)

83. A.L. Cavalcanti de Oliveira, E.R. Bezerra de Mello, Class. Quantum Grav. 23, 5249 (2006)

84. W. Greiner, Relativistic Quantum Mechanics : Wave Equations, 3rd edn. (Springer, Berlin, 2000)

85. H. Asada, T. Futamase, Phys. Rev. D 56, R6062 (1997)

86. H.W. Crater, J.-H. Yoon, C.Y. Wong, Phys. Rev. D 79, 034011 (2009)

87. V.R. Khalil, Phys. Rev. A 71, 012105 (2005)

88. E. R. Figueiredo Medeiros, E. R. B. de Mello, Eur. Phys. J. C 72, 2051 (2012)

89. K. Bakke, C. Furtado, Ann. Phys. (N. Y.) 355, 48 (2015)

90. H.G. Dosch, J.H. Jansen, V.F. Muller, Phys. Norv. 5, 2 (1971)

91. G. Soff, B. Muller, J. Rafelski, W. Greiner, Z. Naturforsch, A 28, 1389 (1973)

92. E. R. Figueiredo Medeiros, E. R. Bezerra de Mello, Eur. Phys. J. C 72, 2051 (2012)
93. M.K. Bahar, F. Yasuk, Adv. HEP 2013, 814985 (2013)

94. L.C.N. Santosa, C.C. Barros Jr., Eur. Phys. J. C 78, 13 (2018)

95. A. Boumali, N. Messai, Can. J. Phys. 92, 1460 (2014)

96. K. Bake, Eur. Phys. J. Plus 127, 82 (2012)

97. K. Bake, Gen. Rel. Grav. 45, 1847 (2013)

98. L.C.N. Santos, C.C. Barros Jr., Eur. Phys. J. C 77, 186 (2017)

99. M. Eshghi, M. Hamzavi, Eur. Phys. J. C 78, 522 (2018)

100. N. Birrell, P. Davies, Quantum Fields in Curved Space (Cambridge University Press, Cambridge, 1984)

101. T. Y. Wu, W. Y. P. Hwang, Relativistic Quantum mechanics and Quantum Fields. World Scientific Publishing Co. Pvt. Ltd., Singapore (1991)

102. F. Ahmed, Commun. Theor. Phys. 68, 735 (2017). arXiv: 1712.01274

103. A.K. Raychaudhuri, S.N.G. Thakurta, Phys. Rev. D 22, 802 (1980)

104. A.F.F. Teixeira, M.J. Reboucas, J.E. Aman, Phys. Rev. D 32, 3309 (1985)

105. M.J. Reboucas, A.F.F. Teixeira, J. Math. Phys. 33, 2885 (1992)

106. G.B. Arfken, H.J. Weber, Mathematical Methods for Physicist (Elsevier Academic Press, New York, 2005)

107. K. Bakke, Ann. Phys. 341, 86 (2014). arXiv:1405.5135 [quant-ph]

108. A. Ronveaux, Heun's Differential Equations (Oxford University Press, Oxford, 1995)

109. A. Vercin, Phys. Lett. B 260, 120 (1991)

110. J. Myrhein, E. Halvorsen, A. Vercin, Phys. Lett. B 278, 171 (1992)

111. K. Bakke, Int. J. Mod. Phys. A 29, 1450117 (2014)

112. M. Eshghi, H. Mehraban, A.I. Ahmadi, Phys. E 94, 106 (2017)

113. M. Eshghi, H. Mehraban, A.I. Ahmadi, EPJ Plus 132, 477 (2017)

114. I. C. Fonseca, K. Bakke, Proc. R. Soc. A 472, 20150362 (2016). arXiv:1606.04725 [quant-ph] 